\title{
TOWARDS A UNIFIED VIEW OF ELECTRON-PHONON COUPLING IN 1D SOLIDS
}

\author{
A. Girlando, A. Painelli
}

Dip. Chim. Gen. Inorg. Anal. e Chim. Fisica, Parma University, Parma, Italy AND Z.G. Soos

Department of Chemistry, Princeton University, Princeton, NJ, USA

\begin{abstract}
We analyze the effect of on-site and on-bond electron-phonon (e-ph) coupling in different classes of quasi one-dimensional (1D) solids in terms of a single model. The model was originally developed to account for e-ph coupling in charge transfer crystals and to interpret their vibrational spectra. The same model is extended to conjugated polymers, showing that important information about the electronic structure can be obtained through a careful analysis of vibrational data. Evidence of e-ph coupling in the spectra of halogen-bridged transition-metal complexes is also presented. The unified model thus applies to different classes of $1 \mathrm{D}$ systems and yields transferable e-ph coupling constants.
\end{abstract}

PACS numbers: 71.38.+i, 71.27.+a, 71.45.Lr

\section{Introduction}

The coupling between electrons and vibrational motions (e-ph coupling) plays a fundamental role in determining the distinctive physical properties of quasi one-dimensional (1D) conducting molecular solids. Although clear and often dramatic signatures of e-ph coupling appear in the vibrational spectra, their analysis is rather difficult due to the large number of vibrational degrees of freedom. Moreover, several and apparently different models have been proposed to interpret e-ph signatures in the spectra of different classes of $1 \mathrm{D}$ solids $[1,2]$. In the present paper we show that the Herzberg-Teller (HT) expansion, developed to account for e-ph spectroscopic effects in charge transfer (CT) crystals [3], can be successfully extended to conjugated polymers and to halogen-bridged transition-metal complexes (MX chains). While providing a unifying framework for current models of linear e-ph coupling, the HT approach quite naturally accounts for quadratic e-ph coupling, essential to define the reference state and to determine sets of transferable e-ph coupling constants. 


\section{Herzberg-Teller expansion}

In the crude adiabatic approximation the electronic Hamiltonian $\left(\mathcal{H}_{\mathrm{e}}\right)$ does not depend on the nuclear normal coordinates $(Q)$. The $Q$-dependence is introduced by expanding $\mathcal{H}_{\mathrm{e}}$ on the $Q$ basis. Consistently with the harmonic approximation, the expansion is carried out up to second order. The e-ph Hamiltonian reads

$$
\mathcal{H}_{\mathrm{e}-\mathrm{ph}}=\sum_{m} \frac{\partial \mathcal{H}_{\mathrm{e}}}{\partial Q_{m}} Q_{m}+\frac{1}{2} \sum_{m, n} \frac{\partial^{2} \mathcal{H}_{\mathrm{e}}}{\partial Q_{m} \partial Q_{n}} Q_{m} Q_{n},
$$

where $m, n$ count the nuclear coordinates. $\mathcal{H}_{\mathrm{e}-\mathrm{ph}}$ acts as a perturbation on the $Q$-independent eigenstates of $\mathcal{H}_{\mathrm{e}}$, yielding $Q$-dependent states. The second derivatives of the ground state energy (the HT nuclear potential) on the $Q$ 's are the force constants for the nuclear motion

$$
\begin{aligned}
\Phi_{m n}= & \Phi_{m n}^{\mathrm{s}}+\left\langle G\left|\frac{\partial^{2} \mathcal{H}_{\mathrm{e}}}{\partial Q_{m} \partial Q_{n}}\right| G\right\rangle \\
& -2 \sum_{m n} \frac{\left\langle G\left|\partial \mathcal{H}_{\mathrm{e}} / \partial Q_{m}\right| F\right\rangle\left\langle F\left|\partial \mathcal{H}_{\mathrm{e}} / \partial Q_{n}\right| G\right\rangle}{E_{F}-E_{G}}
\end{aligned}
$$

where $|G\rangle$ and $|F\rangle$ are the ground and excited states of $\mathcal{H}_{\mathrm{e}}$, with energies $E_{G}$ and $E_{F}$, respectively, and $\Phi_{m n}^{\mathrm{s}}$ represent the skeleton force constants due to the contribution of the core electrons.

The e-ph spectroscopic signatures originate from the third term of Eq. (2), that is from the linear e-ph coupling, which involves low-energy electron fluctuations and is strongly dependent from the electronic excitation spectrum. On the other hand, the contribution from quadratic coupling only involves the ground state, and is conveniently included into a reference force field $\Phi_{m n}^{0}$, which describes the potential due to the core electrons and to the ground state electronic distribution, but does not include the effect of electronic fluctuations.

Let us assume that $\mathcal{H}_{\mathrm{e}}$ is the usual extended Hubbard Hamiltonian. It is convenient to distinguish between phonons modulating site integrals (site energy $\varepsilon$ and electronic repulsion $U$ ), yielding site-diagonal e-ph coupling (e-sph), and phonons modulating intersite integrals (CT integral, $t$, and intersite electron-electron repulsion, $V$ ) yielding bond-diagonal e-ph coupling (e-bph). The interaction between the two types of phonons through the common coupling with the electrons can yield subtle and interesting effects, yet to be explored to their full extent [4]. However, quite often such interaction is prevented by symmetry $[1,3]$, so in many cases it is possible to investigate e-sph and e-bph couplings separately. In CT crystals e-sph has received most attention [1]; in conjugated polymers and MX chains a prevailing role is attributed to $\mathrm{e}-\mathrm{bph}$ and $\mathrm{e}-\mathrm{sph}$ coupling, respectively. In the simplifying hypothesis that just one-electron integrals are modulated by either sph or $\mathrm{bph}$ (i.e. $\varepsilon$ in the case of $\mathrm{sph}$, and $t$ in the case of $\mathrm{bph}$ ), the variation of the force constants due to e-ph coupling (Eq. (2)) has the following special form:

$$
\Phi_{m n}=\Phi_{m n}^{0}-\Delta \Phi_{m n}=\Phi_{m n}^{0}-g_{m} g_{n} \chi
$$

where $g$ is the linear e-ph coupling constant (proportional to the derivative of $\varepsilon$ or $t$ with respect to $Q$ ) and $\chi$ is the relevant electronic susceptibility, either $\chi_{\mathrm{b}}$ and 
$\chi_{\mathrm{v}}$ for e-bph and e-sph coupling, respectively. Equation (3) clearly shows that we can attach quantitative meaning to the linear e-ph coupling constants only if the reference state and frequencies are precisely defined, i.e. if quadratic e-ph coupling is properly accounted for. In the case of e-sph coupling in CT crystals, we have already shown [3] that the reference state is given by the isolated molecular units bearing the same charge they have in the crystal. In CT crystals therefore the reference frequencies are easily obtained from experiment or from empirical correlations, so that the analysis of spectroscopic data yields reliable estimates of e-sph coupling constants. Transferable sets of coupling constants are now available for the main molecular units [1]. On the other hand for conjugated polymers and MX chains the reference state has no experimental counterpart. The problem will be tackled in the following paragraphs.

\section{Polyacetylene: reference state and $\mathrm{e}^{-}-\mathrm{ph}$ coupling constants}

We consider the prototype of conducting polymers, trans-polyacetylene (PA). The spectroscopic effects of e-bph coupling in PA have been considered by many authors [2]. Our analysis combines the linear response approach to e-ph coupling followed in the amplitude mode (AM) model [5] with a molecular spectroscopy approach similar to effective conjugation coordinate (ECC) model [2]. To define the reference state the HT expansion is carried out on the basis of the Wilson's internal coordinates $(R)$ [6]. Assuming $\sigma-\pi$ separability, the reference force field of Eq. (2) can be written as $F^{0}=F^{\sigma}+F^{\pi}$, describing the potential due to $\sigma$-electrons (the core term) and to ground state $\pi$-electron distribution (the quadratic coupling term). The $F$ matrix formulation of HT immediately suggests using standard methods [6] to evaluate the reference frequencies: $F^{0}$ can be transferred from the force field of short polyenes, where the effect of linear e-ph coupling is small due to the large electronic excitation energy. We have constructed $F^{0}$ starting from the butadiene force field [7], changing the diagonal CC stretching force constants to account for the smaller dimerization of PA. The $G$ matrix is defined by the known PA geometry, and the reference frequencies $\omega^{0}$ are obtained by diagonalizing the $G F^{0}$ matrix.

Polyenes and other conjugated hydrocarbons were the motivation for the Hückel theory, which is also the basis of Su-Schrieffer-Heeger (SSH) model of PA [8]. So we start from the Hückel description of PA, with e-bph given by modulating $t$ only. While exploiting the polymer symmetry we factorize the problem. For the relevant $a_{\mathrm{g}}$ subspace, the operator appearing in the linear $\mathrm{e}-\mathrm{ph}$ coupling IIamiltonian (see Eq. (2)) is the staggered bond-order [7], and the corresponding block of the $\Delta \mathcal{F}$ matrix has the form shown in Eq. (3). More precisely three of the $a_{\mathrm{g}}$ modes are coupled to $\pi$-electron fluctuations, while the high energy $\mathrm{CH}$ stretch is practically decoupled. The $a_{\mathrm{g}}$ fundamental frequencies $\omega_{i}$ observed in the Raman spectrum, together with the reference frequencies $\omega_{j}^{0}$ determine the dimensionless e-ph coupling constants $\lambda_{i}=\chi_{\mathrm{b}} g_{i}^{2} / \omega_{i}$ through the solution of a system of $p=3$ linear equations

$$
\sum_{i} \chi_{\mathrm{b}} g_{i}^{2} \prod_{i \neq j}\left\{\left(\omega_{j}^{0}\right)^{2}-\omega_{p}^{2}\right\}=\prod_{i}\left\{\left(\omega_{i}^{0}\right)^{2}-\omega_{p}^{2}\right\}
$$



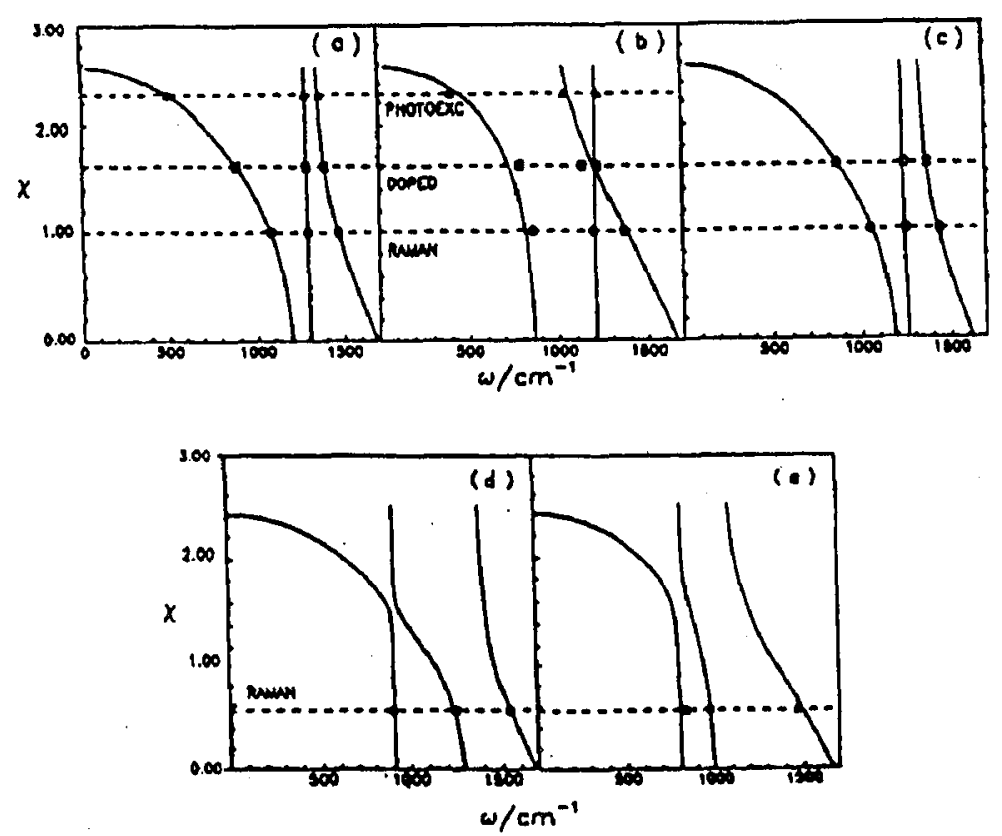

Fig. 1. Relative $\chi_{\mathrm{b}}$ vs. $\omega$ curves, Eqs. (4), for the $a_{\mathrm{g}}$ modes of trans- and cis-PA; (a) $(\mathrm{CH})_{x},(\mathrm{~b})(\mathrm{CD})_{x},(\mathrm{c})\left({ }^{13} \mathrm{CH}\right)_{x},(\mathrm{~d})$ cis- $(\mathrm{CH})_{x},(\mathrm{e})$ cis- $(\mathrm{CD})_{x}$.

TABLE I

Experimental $\Delta \mathcal{F}$ matrix for PA in the $a_{\mathrm{g}}$ symmetry block. Units are $\mathrm{eV} / \AA^{2}$ for stretches, $\mathrm{eV} / \mathrm{rad}^{2}$ for bends, and $\mathrm{eV} / \AA \mathrm{rad}$ for bend-stretches.

\begin{tabular}{c|c|c|r|r|r}
\hline $\begin{array}{c}\text { Internal } \\
\text { coordinate }\end{array}$ & $\begin{array}{c}\text { Symmetry } \\
\text { coordinate }\end{array}$ & $S_{1}$ & $S_{2}$ & \multicolumn{1}{c|}{$S_{4}$} & \multicolumn{1}{c}{$S_{5}$} \\
\hline $\mathrm{C}=\mathrm{C}$ & $S_{1}$ & 8.346 & -7.721 & -1.030 & 0.624 \\
$\mathrm{C}-\mathrm{C}$ & $S_{2}$ & & 7.145 & 0.955 & -0.574 \\
$\mathrm{CCC}$ & $S_{4}$ & & & 0.125 & -0.075 \\
$\mathrm{CCH}$ & $S_{5}$ & & & & 0.044
\end{tabular}

Different choices of $\chi_{\mathrm{b}}$ fit the Raman and dopant- or photo-induced IR frequencies, as shown in Fig. 1a. The $\chi_{\mathrm{b}}=0$ solutions of Eqs. (4) are $\omega_{i}^{0}$, and all modes shift to lower frequencies with increasing $\chi_{\mathrm{b}}$. The $\chi_{\mathrm{b}}=1 \mathrm{fit}$ for the Raman modes of PA is forced, whereas the IR frequencies of doped or photoexcited samples fall on the curves (Fig. 1) with larger $\chi_{b}$. We emphasize that our treatment of linear e-ph coupling is equivalent to AM [5]. However, in our case $\omega_{i}^{0}$ are not freely adjustable parameters, as they have been independently estimated by standard force field methods, on the basis of the second order HT expansion.

Since $\chi_{\mathrm{b}}$ is a purely electronic parameter, it does not vary with isotopic 
substitution. On the other hand, the $a_{\mathrm{g}}$ block of the $\Delta \Phi$ matrix on the $Q$ basis, obtained from the experiment (Eq. (4), can be transformed back to the $\Delta \mathcal{F}$ matrix on the $S$ basis, as shown in Table I. The corresponding e-ph coupling constants on the $S$ basis $(\gamma)$, do not depend on nuclear masses and are expected to be transferable. Figures $1 \mathrm{~b}$ and $1 \mathrm{c}$ clearly demonstrate the transferability of the $\gamma$ 's between isotopically substituted samples. We underline that no additional parameters are needed to fit the PA isotopes. We also tested [9] the transferability of the $\gamma$ 's to cis-polyacetylene (Figs. 1d and 1e). In this case the fit requires a variation of $\chi_{\mathrm{b}}$, reflecting the different electronic structure of the isomers.

To get absolute values of the linear e-ph coupling constants we have to estimate the electronic response $\chi_{\mathrm{b}}$. In the Hückel model $\chi_{\mathrm{b}}$ can be calculated exactly for chains with arbitrary alternation $\delta$. The $\Delta \mathcal{F}$ matrix elements in the $a_{\mathrm{g}}$ subspace for double and single CC symmetry coordinates are [9]

$$
\begin{gathered}
\Delta \mathcal{F}_{\mathrm{dd}}=\chi_{\mathrm{b}} \alpha_{\mathrm{d}}^{2}\left\{\frac{(1+\delta)^{2}}{2}\right\}, \Delta \mathcal{F}_{\mathrm{ss}}=\chi_{\mathrm{b}} \alpha_{\mathrm{s}}^{2}\left\{\frac{(1-\delta)^{2}}{2}\right\}, \\
\Delta \mathcal{F}_{\mathrm{ds}}=\chi_{\mathrm{b}} \alpha_{\mathrm{d}} \alpha_{\mathrm{s}}\left\{\frac{\left(1-\delta^{2}\right)}{2}\right\},
\end{gathered}
$$

where $\alpha_{\mathrm{d} / \mathrm{s}}=\dot{t}^{\prime}\left(R_{\mathrm{d} / \mathrm{s}}\right)$, where $R_{\mathrm{d} / \mathrm{s}}$ is the internal coordinate for double and single band CC stretching. Since for standard PA Hückel parameters $\left(\delta=0.18, t_{0}=\right.$ $2.5 \mathrm{eV}) \chi_{\mathrm{b}}=0.617 \mathrm{eV}^{-1}$, we get $\alpha_{\mathrm{s}}=4.1$ and $\alpha_{\mathrm{d}}=6.4 \mathrm{eV} / \AA$. So linear $t(R)$ with the constant $\alpha$ is a poor approximation, while exponential $t(R)$ implies $\alpha_{\mathrm{d}} / \alpha_{\mathrm{s}}=$ $(1+\delta) /(1-\delta)=1.44$ at $\delta=0.18$, close to the observed ratio 1.56. The Raman and IR active modes of PA thus point to exponential rather than linear $t(R)$. The Hückel model allows one to calculate $\chi_{\mathrm{b}}$ for all wave vectors $k$ in the first Brillouin zone. We can then evaluate the PA force field on the basis of internal coordinates. It turns out [9] that for the infinite polyene the stretch-stretch interactions mediated by the electrons (the "conjugation length" of ECC model [2]) extend over about three unit cells within the Hückel model.

As expected, the strongest e-ph coupling exists for CC stretches (Table I). However, Table I also shows weak coupling of $\pi$-electron fluctuations to CCC and $\mathrm{CCH}$ bends. Coupling to CCC bend is easily understood in terms of Coulomb repulsion $e^{2} / R$. Within $\pi$-electron models (extended Hubbard or Pariser-Parr-Pople, PPP) Coulomb repulsion leads to spin-independent interactions $V\left(R_{p p^{\prime}}\right)$ between sites $p$ and $p^{\prime}$. Modulation of $V$ in addition to $t$ implies that the special form (Eq. (3)) of the $\Delta \mathcal{F}$ matrix is lost. We have performed PPP calculations [10] to disentangle the various contributions. The calculated $\Delta \mathcal{F}$ elements, not scaled for butadiene reference, are $\Delta \mathcal{F}_{\mathrm{dd}}=7.87 ; \Delta \mathcal{F}_{\mathrm{ss}}=7.92 ; \Delta \mathcal{F}_{\mathrm{ds}}=-6.87$; $\Delta \mathcal{F}_{44}(\mathrm{CCC})=0.28$ (the same units as in Table I). It turns out that the PPP parameters, originally developed for $\pi-\pi^{*}$ spectra of small hydrocarbons, lead to good agreement for $\pi$-electron coupling to CCC bends. Potential contributions are consequently discernible in PA vibrations. In the case of CC stretches, such contributions are about one order of magnitude smaller than the $t^{\prime}$ contributions [10], so that $\Delta \mathcal{F}_{\mathrm{ds}}$ does not deviate dramatically from the geometric mean of the diagonal values, as assumed in the Hückel model. We finally remark that also the relative $\chi_{b}$ found for cis-PA with respect to trans-PA (Fig. 1) provides evidence for Coulomb 
interactions. The Hückel model does not distinguish between the two isomers, and the fit to the optical gap would imply $\delta=0.21$ for cis-PA, and a corresponding relative $\chi_{\mathrm{b}}$ of about 0.89 , against the experimental value of 0.56 (Fig. 1).

\section{MX chains: case study for site-diagonal e-ph coupling}

The effects of e-ph coupling on the spectra of MX chains have not been extensively studied so far, even though they are expected to be simpler than in CT crystals or conducting polymers. Limiting attention to the longitudinal vibrations of a typical MX chain as ...Cl- $\mathrm{Pt}^{3+\sigma}-\mathrm{Cl} \mathrm{Pt}^{3-\sigma} \mathrm{Cl}-\mathrm{Pt}^{3+\sigma}-\mathrm{Cl} \ldots(\sigma$ is the amplitude of the site-Charge Density Wave, sCDW), just one mode, the Raman active symmetric $\mathrm{Pt}-\mathrm{Cl}$ stretching, $\omega_{\mathrm{s}}$, is coupled to the electron via the modulation of on-site Pt energy [11]. Thus Eq. (3) has the simple form

$$
\omega_{\mathrm{s}}^{2}=\left(\omega_{\mathrm{s}}^{0}\right)^{2}-g_{\mathrm{s}}^{2} \chi_{\mathrm{v}}
$$

From the discussion in Sec. 2 it is easy to realize that the reference state including the quadratic coupling corresponds to a chain with the same sCDW amplitude of the actual chain, but in which the CT electron fluctuations are negligible. The procedure to estimate $\omega_{\mathbf{s}}^{0}$, is similar to that followed for PA. However, vibrational spectra of metal-halogen systems are far less studied than those of polyenes, and reliable sets of transferable force constants are not available. We have first constructed a force field relevant to isolated $\mathrm{Pt}-\mathrm{Cl}$ complexes in solution, then focused attention on three MX chains: $\left[\mathrm{Pt}(\operatorname{chxn})_{2} \mathrm{Cl}_{2}\right]\left[\mathrm{Pt}(\mathrm{chxn})_{2}\right]\left(\mathrm{ClO}_{4}\right)_{4}$, $\left[\mathrm{Pt}(\mathrm{en})_{2} \mathrm{Cl}_{2}\right]\left[\mathrm{Pt}(\mathrm{en})_{2}\right]\left(\mathrm{ClO}_{4}\right)_{4}$ and $\left[\mathrm{Pt}(\mathrm{chxn})_{2} \mathrm{Cl}_{2}\right]\left[\mathrm{Pt}(\mathrm{chxn})_{2}\right] \mathrm{Cl}_{4}$, hereafter referred to as (1), (2), and (3), respectively. The three compounds exhibit increasing ratios between the $\mathrm{Pt}{ }^{\mathrm{IV}}-\mathrm{Cl}$ and $\mathrm{Pt}^{\mathrm{II}}-\mathrm{Cl}$ distances, $r$. As shown in Table II, the same increasing trend is followed by the frequency of the antisymmetric $\mathrm{Pt}-\mathrm{Cl}$ stretch, $\omega_{\mathrm{a}}$ (IR active), whereas $\omega_{\mathrm{s}}$ decreases. This opposite behavior cannot be easily reproduced by traditional force fields, but can be easily explained as a consequence of e-sph coupling, since $\omega_{\mathrm{s}}$ is coupled to the electron system, whereas $\omega_{\mathrm{a}}$ is not [11]. We have constructed the reference force field for the three systems starting from

TABLE II

Interatomic distances $(\AA)$ along the chain, $\mathrm{Pt}-\mathrm{Cl}$ stretching frequencies $\left(\mathrm{cm}^{-1}\right)$ and SCDW amplitude $(\sigma)$ for the three studied MX solids.

\begin{tabular}{l|c|c|c|c|c|c}
\hline \hline & $d\left(\mathrm{Pt}^{\mathrm{IV}}-\mathrm{Cl}\right)$ & $d\left(\mathrm{Pt}^{\mathrm{II}}-\mathrm{Cl}\right)$ & $\omega_{\mathrm{s}}$ & $\omega_{\mathrm{s}}^{0}$ & $\omega_{\mathrm{a}}$ & $\sigma$ \\
\hline $\mathbf{( 1}^{a}$ & 2.315 & 3.396 & 327 & 335 & 352 & 0.96 \\
$\mathbf{( 2 )}^{b}$ & 2.327 & 3.101 & 312 & 340 & 355 & 0.87 \\
$(3)^{c}$ & 2.324 & 2.834 & 290 & 350 & 362 & 0.78 \\
$a\left[\mathrm{Pt}(\mathrm{chxn})_{2} \mathrm{Cl}_{2}\right]\left[\mathrm{Pt}(\mathrm{chxn})_{2}\right]\left(\mathrm{ClO}_{4}\right)_{4}$, \\
$\left.\quad b \operatorname{Pt}(\mathrm{en})_{2} \mathrm{Cl}_{2}\right]\left[\mathrm{Pt}(\mathrm{en})_{2}\right]\left(\mathrm{ClO}_{4}\right)_{4}$, \\
$\left.\quad c \operatorname{Pt}(\mathrm{chxn})_{2} \mathrm{Cl}_{2}\right]\left[\mathrm{Pt}(\mathrm{chxn})_{2}\right] \mathrm{Cl}_{4}$.
\end{tabular}


the force field of the isolated complexes, adjusting the $\mathrm{Pt}-\mathrm{Cl}$ stretching force constants to account for the actual $\mathrm{Pt}-\mathrm{Cl}$ distances inside the chain and to reproduce the $\omega_{\mathrm{a}}$ frequencies. The reference frequencies $\omega_{\mathrm{s}}^{0}$ obviously increase in the same direction as $\omega_{\mathrm{a}}$, and their difference with respect to the observed $\omega_{\mathrm{s}}$ determines $\chi_{v} g_{s}^{2}$ (Eq. (6)).

In a crystal field-like approach the modulation of $\mathrm{Pt}$ site energies is due to the oscillation of $\mathrm{Cl}^{-}$anions. $g_{\mathrm{s}}$ can be estimated from the variation of the electrostatic potential with $\mathrm{Pt}-\mathrm{Cl}$ stretches [12]: given the $\mathrm{Pt}-\mathrm{Cl}$ distances, $g_{\mathrm{s}}$ turns out to be about $0.2 \mathrm{eV}$ for all the three compounds. To evaluate $\chi_{\mathrm{v}}$ we adopt a simple phenomenological model which accounts for the optical spectra of the studied compounds: the trimer model [11]. Its use is justified by the strong localization of the CT exciton, as demonstrated by the spectroscopic data. Within the trimer model, $\chi_{\mathrm{v}}$ is directly connected to the sCDW amplitude, $\sigma$, and to $t$ as

$$
\chi_{\mathrm{v}}=2\{(1-\sigma) \sigma\}^{3 / 2} / t .
$$

Given $\chi_{\mathrm{v}}$ estimated above, we have adjusted $\sigma$ and $t$ to reproduce the available optical data [13]. The linear connection between $\sigma$ (Table II) and the inverse of the $\mathrm{Pt}-\mathrm{Pt}$ distance, the key parameter controlling the sCDW amplitude [14] gives confidence in the adopted procedure, and puts again in evidence that important information about the electronic structure of $1 \mathrm{D}$ systems can be obtained from a careful analysis of e-ph coupling.

\section{Acknowledgments}

This work was made possible through NATO Collaborative Research Grant No. 900629. Work at Parma was also supported by the National Research Council (CNR) and by the Ministry of the University and Scientific and Technological Research (MURST), and at Princeton by the National Science Foundation through NSF-DMR-9300163.

\section{References}

[1] C. Pecile, A. Painelli, A. Girlando, Mol. Cryst. Liq. Cryst. 171, 69 (1989) and references therein.

[2] M. Gussoni, C. Castiglioni, G. Zerbi, in: Spectroscopy of Advanced Materials, Eds. R.J.H. Clark, R.E. Hester, Wiley, New York 1991, p. 251 and references therein.

[3] A. Painelli, A. Girlando, J. Chem. Phys. 84, 5655 (1986).

[4] A. Painelli, A. Girlando, Solid State Comm. 63, 1087 (1987).

[5] B. Horovitz, Solid State Comm. 41, 729 (1982).

[6] E.B. Wilson, J.C. Decius, P. Cross, Molecular Vibrations, McGraw-Hill, New York 1955.

[7] A. Girlando, A. Painelli, Z.G. Soos, Chem. Phys. Lett. 198, 9 (1992); J. Chem. Phys. 98, 7459 (1993).

[8] W.P. Su, J.R. Schrieffer, A.J. Heeger, Phys. Rev. Lett. 44, 1698 (1979).

[9] A. Girlando, A. Painelli, G.W. Hayden, Z.G. Soos, Chem. Phys. 184, 139 (1994).

[10] Z.G. Soos, G.W. Hayden, A. Girlando, A. Painelli, J. Chem. Phys. 100, 7144 (1994).

[11] A. Girlando, A. Painelli, Synth. Met. 41, 2721 (1991). 
[12] A. Painelli, A. Girlando, Synth. Met. 29, F181 (1989).

[13] H. Okamoto, T. Mitani, K. Toriumi, M. Yamashita, Met. Sci. Eng. B 13, L9 (1992).

[14] M. Aluani, J.W. Wilkins, R.C. Albers, J.M. Wills, Phys. Rev. Lett. 71, 1415 (1993). 